\title{
Effect of the Solvent on the Oxygen Evolution Reaction at the $\mathrm{TiO}_{2}$-Water Interface
}

\author{
Patrick Gono, ${ }^{*}$ Francesco Ambrosio, and Alfredo Pasquarello \\ Chaire de Simulation à l'Echelle Atomique (CSEA), Ecole Polytechnique Fédérale de \\ Lausanne (EPFL), CH-1015 Lausanne, Switzerland \\ E-mail: patrick.gono@epfl.ch
}




\begin{abstract}
We investigate the solvation effect of water on the overpotentials of the oxygen evolution reaction on rutile $\mathrm{TiO}_{2}$ by applying the thermodynamic integration method on atomistic model interfaces with and without the water molecules. We compare the results at the vacuum interface with the commonly used computational hydrogen electrode method, finding overall good agreement. The effect of the solvent is found to be twofold. First, the explicit treatment of the solvent can lead to equilibrium configurations differing from the relaxed structures without solvent. Second, the overpotentials can be affected by up to $0.5 \mathrm{eV}$. The energetics are subject to electrostatic effects at the interface rather than to modifications in the hydrogen bond network. These results provide a promising perspective on the use of implicit models for treating the solvent.
\end{abstract}

\title{
1 Introduction
}

The oxygen evolution reaction (OER) has been extensively studied for its role in photoelectrocatalytic water splitting for hydrogen production. ${ }^{1-6}$ Early on, $\mathrm{TiO}_{2}$ has been identified as a possible catalyst. ${ }^{7}$ The mechanism of the OER on this material, and especially its rutile phase, has attracted a vast body of research. ${ }^{8-23}$ It has been proposed that in acidic conditions the reaction on rutile takes place through four proton-coupled electron transfer (PCET) steps: ${ }^{24}$

$$
\begin{gathered}
{ }^{*}+\mathrm{H}_{2} \mathrm{O} \longrightarrow \mathrm{OH}_{\mathrm{ads}}+\mathrm{H}^{+}+e^{-}, \\
\mathrm{OH}_{\mathrm{ads}} \longrightarrow \mathrm{O}_{\mathrm{ads}}+\mathrm{H}^{+}+e^{-}, \\
\mathrm{O}_{\mathrm{ads}}+\mathrm{H}_{2} \mathrm{O} \longrightarrow \mathrm{OOH}_{\mathrm{ads}}+\mathrm{H}^{+}+e^{-}, \\
\mathrm{OOH}_{\mathrm{ads}} \longrightarrow{ }^{*}+\mathrm{O}_{2 \mathrm{~g}}+\mathrm{H}^{+}+e^{-},
\end{gathered}
$$

where the asterisk represents an active site on the catalyst on which the subsequent intermediate species are adsorbed. The OER has also been modeled in the literature through the consideration of other mechanisms or intermediates, ${ }^{25,26}$ but the one given above is by far the most widely adopted. ${ }^{24,27}$ The computational hydrogen electrode (CHE) method, developed 
by Nørskov et al. ${ }^{28}$ allows one to approximate the free energy differences corresponding to these four PCET steps. In the framework of this method, density functional theory (DFT) is used to compute the binding energies of the various reaction intermediates at the electrode/electrolyte interface. These binding energies are then augmented by thermodynamic corrections, such as the zero point energy of lattice vibrations and entropy contributions. While reasonably successful at predicting the overall reaction overpotential of the OER on $\mathrm{TiO}_{2},{ }^{24}$ the CHE method suffers from two drawbacks. First, entropic effects are included only in an approximate fashion. Second, solvation effects are disregarded and thus their role remains elusive.

The solvation effect is multi-faceted in nature. For one thing, the solvent can stabilize or destabilize the adsorbate, changing the binding energies of the reaction intermediates. ${ }^{29}$ Another important role of the solvent lies in the complex way it affects the entropy contributions to the free energy differences. Furthermore, one has to take the $\mathrm{pH}$ of the electrolyte at the solid interface into consideration to describe the type of surface and its coverage under OER conditions. ${ }^{30}$

Various schemes have been employed to quantify the effects of the solvent. The roughest approximation ignores the solvent completely. ${ }^{31}$ The assumption here is that the solvent affects all the intermediates nearly equally, and thus the solvation effect cancels out in the free energy differences considered. Implicit solvation methods approximate the solvent through a continuum model based on electrostatics. ${ }^{13,32-34}$ Although this approach works well for long range effects, the method cannot account for the immediate vicinity of an adsorbate in contact with a protic solvent such as liquid water, which interacts significantly with solutes through hydrogen bonds and van der Waals interactions. Furthermore, in this case, it is difficult to distinguish the effect of the solvent from the adopted implicit approximation. Explicit solvation methods can employ one or several layers of water molecules in a DFT calculation to compute the binding energies of adsorbates. These water layers are placed above the surface and can be ice-like or undergo structural relaxation. ${ }^{29,35-39}$ In this ap- 
proach, the solvation effect amounts to a straightforward correction to the binding energies of the reaction intermediates. If the water layer is allowed to relax, a doubt remains whether the optimized water structure is an accurate representation of the liquid at the solvated interface. $^{37}$ In any case, DFT structural relaxations are performed at $0 \mathrm{~K}$, and therefore such schemes fail in describing the finite temperature behavior of liquid solvents. Furthermore, relaxation of the water layer near the adsorbate can lead to an overestimate of the stabilization due to hydrogen bonds, some of which can be disrupted in finite temperature dynamics where disorder is taken into account. ${ }^{37}$ More sophisticated modeling of the solvent involves molecular dynamics (MD) simulations, which sample multiple local minima of the geometry by simulating the finite temperature behavior of the system from first principles. In conjunction with thermodynamic integration (TI), ${ }^{40}$ such MD simulations enable one to compute the free energy of a reaction in the presence of an explicit solvent. ${ }^{25,41,42}$ In particular, Cheng et al. applied such a scheme to some of the PCET steps of the oxygen evolution reaction at the $\mathrm{TiO}_{2}$-water interface. ${ }^{25}$ However, there was no attempt to isolate the effect of the solvent, and it thus remains unclear whether this high-level modeling improves the description of the reaction with respect to simpler approaches, such as the CHE method.

In this work, we aim at evaluating the role of the solvent in determining the free energies of the OER steps. For this, we develop a formulation in which thermodynamic integration and molecular dynamics are combined. The effect of the solvent is determined by modeling the PCET steps of the OER at the rutile $\mathrm{TiO}_{2}$ surface in the presence and absence of explicit water molecules. We also evaluate the free energies of the four PCET steps with the computational hydrogen electrode method, ${ }^{28}$ in which the solvent is neglected and thermal effects are treated in an approximate fashion. Such an approach allows us to isolate the effect of the solvent on the energetics of the full OER path. This study quantifies the role of the solvent in determining OER overpotentials and sets a benchmark for more approximative schemes. 


\section{Methods}

\subsection{Computational Details}

All the simulations are carried out with the CP2K suite of codes. ${ }^{43}$ The results are computed at the semilocal level of theory using the rVV10 functional, ${ }^{44}$ which accounts for nonlocal van der Waals interactions, and has been adjusted to give an accurate description of the density and the structural properties of liquid water by setting the $b$ parameter to $9.3{ }^{45,46}$ We remark that more advanced levels of theory are necessary to allow for accurate comparison between calculated and measured energies. ${ }^{47,48}$ However, the semilocal level of theory is sufficient to establish the effect of the solvent on the free energy of the reaction and is therefore used in this work for its lower computational cost. Double- $\zeta$ MOLOPT basis sets are used for titanium, triple- $\zeta$ cc-TZ basis sets for oxygen and hydrogen. The cutoff for the density is set to 500 Ry. We use Goedecker-Teter-Hutter pseudopotentials. ${ }^{49}$ The molecular dynamics simulations employ a time step of 0.5 fs. A Nosé-Hoover thermostat regulates the temperature, which is set to $350 \mathrm{~K}$ in order to ensure proper diffusive behavior of liquid water. ${ }^{50}$ Through the CHE method, we estimate that using this temperature rather than room temperature has a negligible effect on the calculated energies (less than $0.06 \mathrm{eV}$ ).

\subsection{Thermodynamic Integration Method}

We express the free energy difference corresponding to a proton-coupled electron transfer step as:

$$
\Delta G=G[P]-G[R]+\mu\left[\mathrm{H}^{+}\right]+\mu\left[e^{-}\right]
$$

Adopting the scale of the reversible hydrogen electrode, we have that: ${ }^{51}$

$$
\mu\left[\mathrm{H}^{+}\right]+\mu\left[e^{-}\right]=\frac{1}{2} \mu\left[\mathrm{H}_{2}\right]
$$


We define the free energy difference between the product $P$ and the reactant $R$ as:

$$
G[P]-G[R]=\Delta_{\mathrm{dh}} A+\Delta_{\mathrm{zp}} E,
$$

where $\Delta_{\mathrm{dh}} A$ is the dehydrogenation free energy integral, and $\Delta_{\mathrm{zp}} E$ is the zero point energy difference between the product and reactant systems. The dehydrogenation free energy integral of the reactant species is calculated through thermodynamic integration of the vertical dehydrogenation energies $\left\langle\Delta_{\mathrm{dh}} E\right\rangle_{\eta}:{ }^{41}$

$$
\Delta_{\mathrm{dh}} A=\int_{0}^{1}\left\langle\Delta_{\mathrm{dh}} E\right\rangle_{\eta} \mathrm{d} \eta
$$

The zero point energy difference $\Delta_{\mathrm{zp}} E$ is computed as the difference of the vibrational zero point energies corresponding to the adsorbed product and reactant species. The vibrational analysis is carried out in the absence of water using the harmonic approximation. The vibrational spectrum of the slab is assumed to be unperturbed by the adsorbate. Finally, the free energy (2) is obtained as:

$$
\Delta G=\Delta_{\mathrm{dh}} A+\frac{1}{2} \mu\left[\mathrm{H}_{2}\right]+\Delta_{\mathrm{zp}} E
$$

We run MD simulations of the systems with the various intermediates for at least 4 ps in order to obtain properly averaged structural properties and vertical dehydrogenation energies. In the case of $\mathrm{OOH}_{\text {ads }}$ an harmonic constraint has been used to restrain the hydrogen in the $\mathrm{OOH}$ adsorbate, as it has the tendency to spontaneously dissociate and bond with a nearby bridge oxygen atom at the surface. Hence, the intermediate considered in this case corresponds to a metastable configuration. Since the constraints are applied both in the reactant and in the product, their effects on the vertical dehydrogenation energies cancel to a large extent and can be neglected. ${ }^{46,52}$

For every value of the Kirkwood mixing parameter $\eta$, the dynamics is evolved according 
to a linear combination of the forces achieved with and without the hydrogen atom. The vertical dehydrogenation energy level is calculated as the difference between the total energy of a configuration of the reactant and that of the same configuration with the hydrogen atom removed. For $\eta=1$, the hydrogen atom is added in a configuration of the product and allowed to relax, with the rest of the structure kept fixed. For intermediate values of $\eta(0<\eta<1)$, we find that MD runs starting from equilibrated configurations of the reactant (i.e. with the

hydrogen atom present) yield converged vertical dehydrogenation energies within about 1 ps. The statistical error on the average vertical dehydrogenation energy is estimated using a blocking analysis. ${ }^{53}$ We find that the error incurred by the application of the trapezoidal integration rule is lower than the statistical error in all cases.

\subsection{Thermodynamic Corrections}

Table 1: Zero point energies $E_{\mathrm{zp}}$, the corrections due to entropy $T S$, and the internal energy $\Delta U^{0 \rightarrow T}$ for the various free and adsorbed species. All values are in $\mathrm{eV}$.

\begin{tabular}{lccc}
\hline \hline Species: & $E_{\text {zp }}$ & $T S$ & $\Delta U^{0 \rightarrow T}$ \\
\hline $\mathrm{H}_{2}$ & 0.265 & 0.434 & 0.103 \\
$\mathrm{H}_{2} \mathrm{O}$ & 0.573 & 0.673 & 0.103 \\
$\mathrm{O}_{2}$ & 0.092 & 0.606 & 0.103 \\
$\mathrm{H}_{2} \mathrm{O}_{\text {ads }}$ & 0.691 & 0.099 & 0.060 \\
$\mathrm{OH}_{\text {ads }}$ & 0.356 & 0.078 & 0.046 \\
$\mathrm{O}_{\text {ads }}$ & 0.092 & 0.027 & 0.019 \\
$\mathrm{H}_{2} \mathrm{O}_{2}$ ads & 0.789 & 0.136 & 0.080 \\
$\mathrm{OOH}_{\text {ads }}$ & 0.473 & 0.149 & 0.082 \\
$\mathrm{O}_{2}$ ads & 0.147 & 0.159 & 0.082 \\
\hline \hline
\end{tabular}

The thermodynamic corrections, including the zero point energy, the entropy, and the temperature dependence of the internal energy, are calculated in the harmonic approximation from the vibrational spectrum of the adsorbed species at the interface with vacuum. ${ }^{48}$ The corrections for liquid and gaseous species are calculated using a simulation cell containing a single molecule. For the non-adsorbed species, the rotational and translational entropy are 
also accounted for. In the case of liquid water, a fugacity of $3534 \mathrm{~Pa}$ is used to compute the translational entropy. ${ }^{29} \mathrm{~A}$ temperature of $298.15 \mathrm{~K}$ is used. The thermodynamic corrections used in the free energy calculations are listed in Table 1.

\subsection{Blue Moon Simulations}

The Blue Moon method allows us to determine the free energy profile between an initial and final state of the same system, which are separated by a free-energy barrier. ${ }^{56,57}$ In this work, we apply this method to the reaction:

$$
\mathrm{O}_{\text {ads }}+\mathrm{H}_{2} \mathrm{O} \rightarrow \mathrm{H}_{2} \mathrm{O}_{2} \text { ads }
$$

which corresponds to the formation of $\mathrm{H}_{2} \mathrm{O}_{2}$ ads through the capture of a water molecule of the liquid by $\mathrm{O}_{\text {ads }}$. The $\mathrm{O}-\mathrm{O}$ distance between the adsorbed oxygen atom and the oxygen atom of a coordinated water molecule is taken as the reaction coordinate $\xi$. We choose seven values of the $\mathrm{O}-\mathrm{O}$ distance spanning the range between the equilibrium distance in the initial $\left(\xi_{0}=2.69 \AA\right)$ and final configurations $\left(\xi_{1}=1.49 \AA\right)$. For each value, the distance between the two atoms is fixed and a constrained MD simulation is carried out. The force acting on the constraint is then computed for each value of the reaction coordinate as the average of the corresponding Lagrange coefficient $\langle\lambda\rangle_{\xi}$. MD simulations with durations of 1 ps are found to be sufficient to achieve accurate results. The free energy profile of the reaction is then calculated as the integral of these forces:

$$
\Delta F=\int_{\xi_{0}}^{\xi_{1}} \mathrm{~d} \xi\langle\lambda\rangle_{\xi} .
$$

The statistical errors are estimated via a blocking analysis. ${ }^{53}$ The error estimate associated with the trapezoidal integration rule is lower than the statistical uncertainty. 


\section{Results}
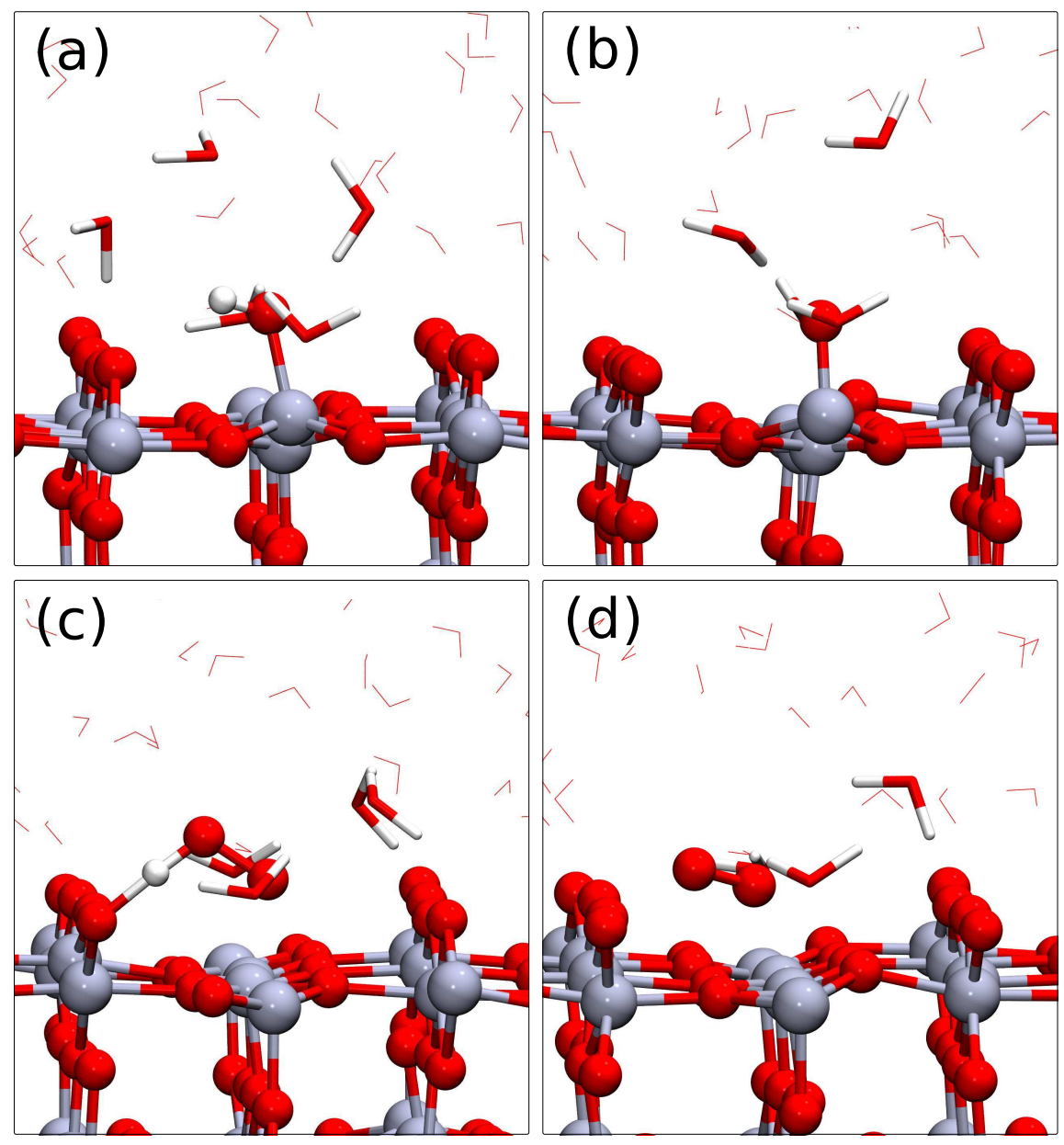

Figure 1: Detailed views of the first solvation shell for (a) $\mathrm{OH}_{\text {ads }}$, (b) $\mathrm{O}_{\text {ads }}$, (c) $\mathrm{OOH}_{\mathrm{ads}}$, and (d) $\mathrm{O}_{2}$ ads. White atoms represent hydrogen, red atoms oxygen, and gray atoms titanium. For clarity, water molecules far away from the active site are rendered schematically.

The rutile $\mathrm{TiO}_{2}(110)$ surface is modeled by a 5 -layer slab exposing a $4 \times 2$ interface repeat unit in the supercell. The slab is separated from its nearest periodic image in the $z$ direction by $15 \AA$. In the solvated case, this region is filled with 80 water molecules. We consider all reaction intermediates to be adsorbed at a single five-coordinated surface titanium atom (Fig. 1). In particular, the MD simulation of the $\mathrm{O}_{\text {ads }}$ adsorbate in the presence of explicit water indicates that this species forms a single bond with the surface through the Ti atom. However, at the vacuum interface $\mathrm{O}_{\text {ads }}$ forms an additional bond with a surface $\mathrm{O}$ atom in its most stable configuration. Therefore, to enable a meaningful comparison with the 
solvated case, we sample the vacuum interface with $\mathrm{O}_{\text {ads }}$ in a similar configuration without extra bonds, which is found to correspond to a local minimum. In the present work, polaron formation at this interface ${ }^{48}$ is disregarded as the focus is on the solvent.

\subsection{Solvent effect on individual PCET steps}

To calculate the relevant free energy differences corresponding to the four PCET steps of eq. (1), we start by considering the following reactions:

$$
\begin{gathered}
\mathrm{H}_{2} \mathrm{O}_{\mathrm{ads}} \longrightarrow \mathrm{OH}_{\mathrm{ads}}+\mathrm{H}^{+}+e^{-}, \\
\mathrm{OH}_{\mathrm{ads}} \longrightarrow \mathrm{O}_{\mathrm{ads}}+\mathrm{H}^{+}+e^{-}, \\
\mathrm{H}_{2} \mathrm{O}_{2 \text { ads }} \longrightarrow \mathrm{OOH}_{\mathrm{ads}}+\mathrm{H}^{+}+e^{-}, \\
\mathrm{OOH}_{\mathrm{ads}} \longrightarrow \mathrm{O}_{2 \text { ads }}+\mathrm{H}^{+}+e^{-} .
\end{gathered}
$$

We remark that the first reaction starts from an initial water molecule that is adsorbed on the surface. Likewise, the final oxygen molecule in the products of the last reaction remains attached to the surface. Furthermore, the third reaction step considered in eq. (1) (formation of $\mathrm{OOH}_{\text {ads }}$ ) proceeds from $\mathrm{H}_{2} \mathrm{O}_{2}$ ads . This set of reactions ensures that each PCET is a reaction of the type $\mathrm{XH}_{\mathrm{ads}} \rightarrow \mathrm{X}_{\mathrm{ads}}+\mathrm{H}^{+}+e^{-}$, where the reactant and product species are both adsorbed at the interface and only differ by a single hydrogen atom.

Following the procedure outlined in the sec. 2.2, we write the free energy differences corresponding to the four PCET steps in eq. (9) as:

$$
\begin{aligned}
& \Delta G_{1}^{\prime}=\Delta_{\mathrm{dh}} A_{\mathrm{H}_{2} \mathrm{O} \text { ads }}+\frac{1}{2} \mu\left[\mathrm{H}_{2}\right]+\Delta_{\mathrm{zp}} E_{\mathrm{H}_{2} \mathrm{O} \text { ads }}, \\
& \Delta G_{2}^{\prime}=\Delta_{\mathrm{dh}} A_{\mathrm{OH} \text { ads }}+\frac{1}{2} \mu\left[\mathrm{H}_{2}\right]+\Delta_{\mathrm{zp}} E_{\mathrm{OH} \text { ads }}, \\
& \Delta G_{3}^{\prime}=\Delta_{\mathrm{dh}} A_{\mathrm{H}_{2} \mathrm{O}_{2} \text { ads }}+\frac{1}{2} \mu\left[\mathrm{H}_{2}\right]+\Delta_{\mathrm{zp}} E_{\mathrm{H}_{2} \mathrm{O}_{2} \text { ads }}, \\
& \Delta G_{4}^{\prime}=\Delta_{\mathrm{dh}} A_{\mathrm{OOH} \text { ads }}+\frac{1}{2} \mu\left[\mathrm{H}_{2}\right]+\Delta_{\mathrm{zp}} E_{\mathrm{OOH} \text { ads }},
\end{aligned}
$$

where $\Delta_{\mathrm{dh}} A_{X \text { ads }}$ is the dehydrogenation free energy of species $X$ calculated via the TI 
method, $\Delta_{\mathrm{zp}} E_{X \text { ads }}$ is the zero point energy difference between the reactant and the dehydrogenated product, and the chemical potential of the hydrogen molecule $\mu\left[\mathrm{H}_{2}\right]$ is calculated in the gas phase approximation at the conditions of the reversible hydrogen electrode. ${ }^{46}$ Note that the calculated $\Delta G^{\prime}$ values are not to be directly compared with the free energies computed by, e.g., Valdés et al., ${ }^{24}$ as the initial and final configurations of eq. (9) contain adsorbed rather than liquid and gaseous species, respectively. These free energy steps are calculated in the same framework both in the presence and in the absence of the explicit solvent. This approach makes it possible to isolate the effect of the solvent on the energetics of the OER. The same values are also computed without solvent using the CHE method. The calculated zero point energies and all the thermodynamic corrections used in the CHE approach are given in sec. 2.3.
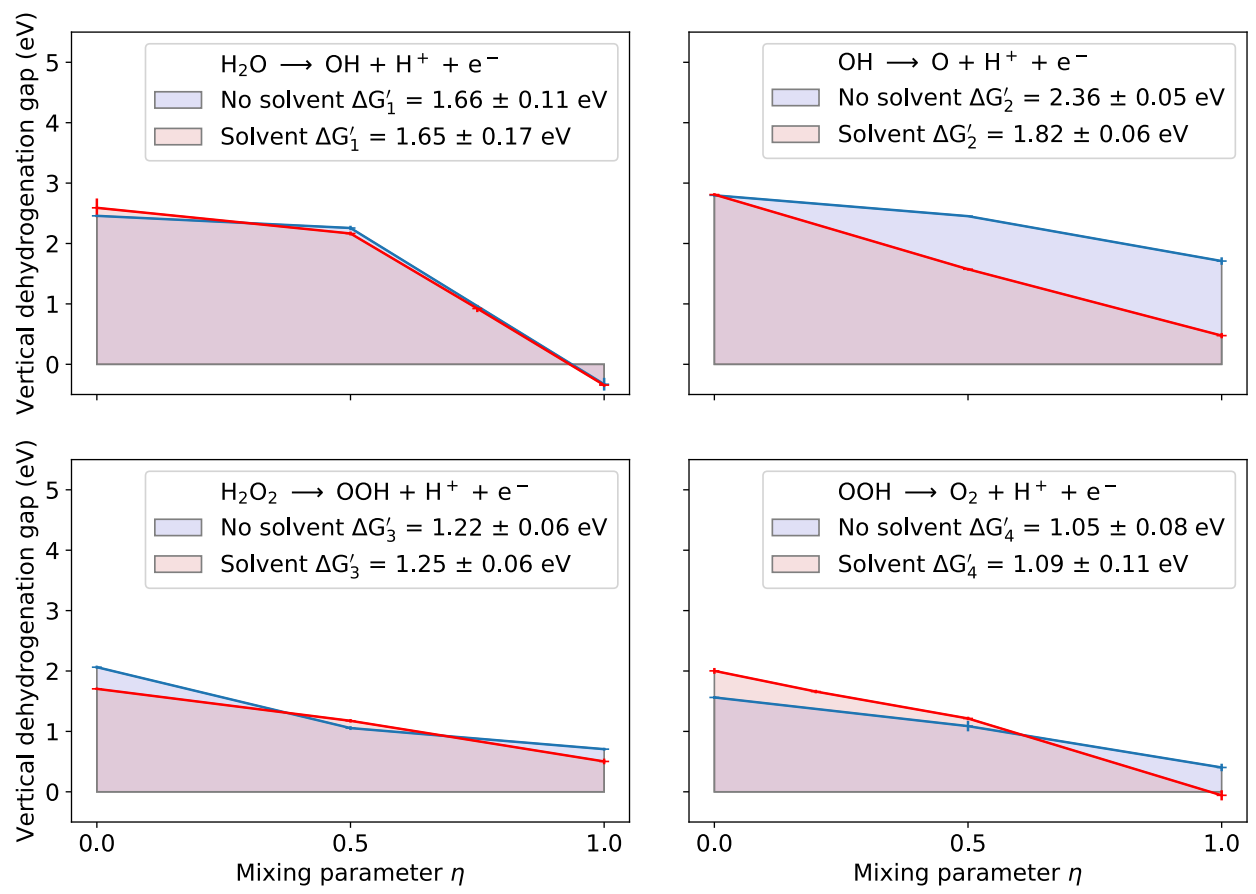

Figure 2: Vertical dehydrogenation energies calculated for the four reactions in eq. (1), including the zero point energy correction and the chemical potential of hydrogen, as a function of the Kirkwood mixing parameter $\eta$. The area under the curve represents the free energy corresponding to the proton-coupled electron transfer step.

The free energies obtained with the CHE and the TI simulations are given in Table 2. In 
particular, the results from the TI simulations are inferred from Fig. 2, where the areas underneath the curves represent the free energy steps $\Delta G^{\prime}$ in both the presence and absence of water. We first compare the free energy values calculated with the CHE approach at the vacuum interface with the TI results without solvent. These energies differ by less than $0.1 \mathrm{eV}$, except for the last step, where small variations in the binding energies and in the thermodynamic corrections lead to a slightly larger difference of $0.34 \mathrm{eV}$. Overall, this indicates that the CHE model is properly accounting for thermal effects. Next, we focus on the effect of the solvent by comparing the TI results obtained with and without the explicit water. The free energy differences $\Delta G_{1}^{\prime}, \Delta G_{3}^{\prime}$, and $\Delta G_{4}^{\prime}$ are all barely affected by the inclusion of the solvent, with differences below $0.1 \mathrm{eV}$. At variance, for the second reaction step, the difference in $\Delta G_{2}^{\prime}$ is more sizable and amounts to $0.54 \mathrm{eV}$. Hence, this comparison shows that the inclusion of the water has a significant effect only on the second PCET reaction.

Table 2: Free energy steps $\Delta G_{i}^{\prime}$ calculated using the thermodynamic integration (TI) method with and without solvent, and the computational hydrogen electrode (CHE) method. All values are in $\mathrm{eV}$.

\begin{tabular}{cccc}
\hline \hline & CHE & TI no solvent & TI solvent \\
\hline$\Delta G_{1}^{\prime}$ & 1.76 & $1.66 \pm 0.11$ & $1.65 \pm 0.17$ \\
$\Delta G_{2}^{\prime}$ & 2.43 & $2.36 \pm 0.05$ & $1.82 \pm 0.06$ \\
$\Delta G_{3}^{\prime}$ & 1.12 & $1.22 \pm 0.06$ & $1.25 \pm 0.06$ \\
$\Delta G_{4}^{\prime}$ & 1.38 & $1.05 \pm 0.08$ & $1.09 \pm 0.11$ \\
\hline \hline
\end{tabular}

In order to get deeper insight into the solvent effect, we analyze in detail the structural features of the adsorbate in the presence of the solvent, most notably the number of hydrogen bonds. Table 3 gives the number of coordinated water molecules and hydrogen bonds associated with the oxygen atoms of the adsorbed reaction intermediate. The number of water molecules coordinated with the adsorbate is obtained as the average number of oxygen atoms belonging to water molecules that are closer than $3.5 \AA$. Following a commonly adopted criterion in the literature, ${ }^{45,46,54}$ we assume that an accepted hydrogen bond is formed when 
Table 3: For each adsorbate, we give the average number of coordinated water molecules (cw), the sum of accepted $(\mathrm{aH})$ and donated $(\mathrm{dH})$ hydrogen bonds, and the variation of bond length between the surface Ti atom and the $\mathrm{O}$ atom of the adsorbate $\left(\Delta_{\mathrm{Ti}-\mathrm{O}}\right)$ upon inclusion of the solvent.

\begin{tabular}{lccc}
\hline \hline Adsorbate & $\mathrm{cw}$ & $\mathrm{aH}+\mathrm{dH}$ & $\Delta_{\text {Ti-O }}(\AA)$ \\
\hline $\mathrm{H}_{2} \mathrm{O}_{\text {ads }}$ & 5.71 & 2.44 & 0.04 \\
$\mathrm{OH}_{\text {ads }}$ & 5.53 & 1.69 & 0.04 \\
$\mathrm{O}_{\text {ads }}$ & 5.65 & 2.13 & -0.20 \\
$\mathrm{H}_{2} \mathrm{O}_{2}$ ads & 6.44 & 4.26 & 0.29 \\
$\mathrm{OOH}_{\text {ads }}$ & 4.72 & 1.85 & 0.21 \\
$\mathrm{O}_{2}$ ads & 4.08 & 1.35 & -0.11 \\
\hline \hline
\end{tabular}

the $\mathrm{OHO}$ angle involving an $\mathrm{O}$ atom of a coordinated water molecule, one of its hydrogen atoms, and an oxygen atom of the adsorbate is greater than $140^{\circ}$. We consider the hydrogen bond to be donated when it involves a hydrogen atom of the adsorbate and a water molecule.

Regarding the first OER step, we need to consider $\mathrm{H}_{2} \mathrm{O}_{\text {ads }}$ and $\mathrm{OH}_{\text {ads }}$. In the case of the surface covered by water, the adsorbed water molecule coordinates on average with 5.71 water molecules. It temporarily accepts one hydrogen bond, whereas it donates its hydrogen atoms to form up to two hydrogen bonds with neighboring water molecules of the liquid. In total, 2.44 hydrogen bonds are formed. $\mathrm{OH}_{\mathrm{ads}}$ coordinates a similar number of water molecules (5.53), and forms 1.69 hydrogen bonds [cf. Fig. 1(a)]. While the lower number of hydrogen bonds in the product system suggests an increase of the free energy step $\Delta G_{1}^{\prime}$ upon inclusion of the solvent, the calculated value is found to remain unaffected. In the second reaction, the product $\mathrm{O}_{\text {ads }}[\mathrm{cf}$. Fig. 1(b)] forms 2.13 hydrogen bonds on average, more than the 1.69 formed by the reactant $\mathrm{OH}_{\text {ads }}$. The presence of more hydrogen bonds among the products suggests greater stabilization in line with the decrease in $\Delta G_{2}^{\prime}$ observed upon solvation. Focusing on the third step, we find that the reactant $\mathrm{H}_{2} \mathrm{O}_{2}$ ads coordinates a large number of water molecules (6.44) and is stabilized by 4.26 hydrogen bonds on average. Both oxygen atoms of the adsorbate accept one hydrogen bond, and both hydrogen atoms donate one. For the product $\mathrm{OOH}_{\text {ads }}$ the number of hydrogen bonds drops to 1.85 [cf. Fig. 1(c)]. 
This weaker hydrogen-bond stabilization of the product as compared to the reactant hints at a larger value of $\Delta G_{3}^{\prime}$ upon solvation. However, no significant increase of $\Delta G_{3}^{\prime}$ is observed. In the last reaction, both oxygen atoms of the product $\mathrm{O}_{2}$ ads $[\mathrm{cf}$. Fig. $1(\mathrm{~d})]$ form on average less than a single hydrogen bond with nearby water molecules, leading to a hydrogen bond number of 1.35 . This results in a weaker hydrogen bond stabilization of the product than of the reactant $\mathrm{OOH}_{\mathrm{ads}}$. On this basis, the free energy difference $\Delta G_{4}^{\prime}$ is expected to be larger in the presence of the solvent than at the vacuum interface. However, the values of $\Delta G_{4}^{\prime}$ are found to be virtually identical in both cases. Hence, we conclude that the analysis based on hydrogen-bond counting is insufficient to explain the observed trends in free energy.

(a)

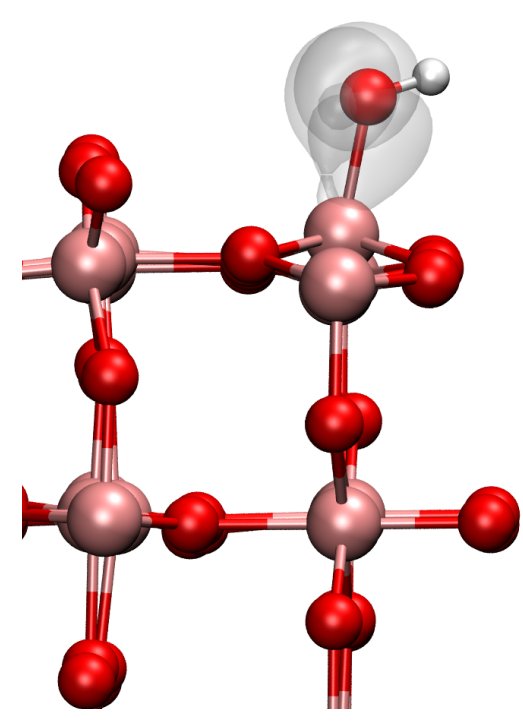

(b)

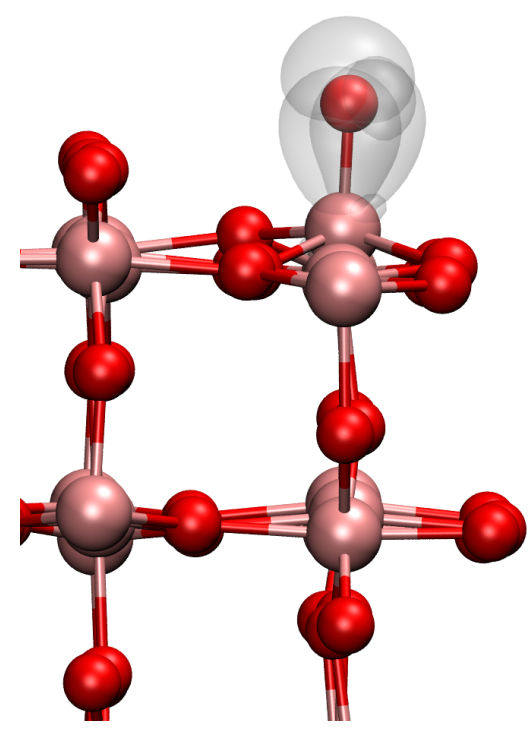

Figure 3: Centers of maximally localized Wannier functions (blue balls) in the case of (a) $\mathrm{OH}_{\mathrm{ads}}$ and (b) $\mathrm{O}_{\mathrm{ads}}$. In both cases, the oxygen atom of the adsorbate carries eight Wannier centers, but Wannier centers of opposite spin almost coincide and thus appear as a single ball. Since an extra proton is present in the case of $\mathrm{OH}_{\mathrm{ads}}$, the adsorbates $\mathrm{OH}_{\mathrm{ads}}$ and $\mathrm{O}_{\mathrm{ads}}$ are effectively in a charge state of -1 and -2 , respectively. The isosurfaces illustrate the shape of the Wannier orbitals.

A different explanation must be found for the large solvent stabilization of $\mathrm{O}_{\text {ads }}$ involved in the second OER step. By studying the Wannier functions associated with the relaxed OER intermediates, we find that the electronegative $\mathrm{O}_{\text {ads }}$ acquires two electrons from the $\mathrm{TiO}_{2}$ slab 
to fill its shell (see Fig. 3). This is in contrast with $\mathrm{OH}_{\text {ads }}$ and $\mathrm{OOH}_{\text {ads }}$, both of which only attract one electron. The larger partial negative charge located at $\mathrm{O}_{\text {ads }}$ could then lead to a different electrostatic stabilization in vacuum $(\epsilon=1)$ and in water $(\epsilon \approx 80)$. To investigate this effect, we carry out two tests. First, the adsorbed oxygen atom at the solvated and at the vacuum interface is replaced by a fluorine atom. The fluorine atom requires only a single electron to fill its shell, leading to a different partial charge at the interface. The configuration of the system is kept fixed upon fluorine substitution. In agreement with our assumption, we find that the solvent stabilization of $\mathrm{F}_{\text {ads }}$ is reduced with respect to that of $\mathrm{O}_{\text {ads }}$ by as much as $1.4 \mathrm{eV}$. The second test entails a classical electrostatics simulation of a Gaussian charge distribution at a dielectric interface modeling the periodic DFT computational cell. ${ }^{55}$ We find that the electrostatic energy difference between a system with a charge $q=-e$ and $q=-2 e$ is approximately $1.5 \mathrm{eV}$. The magnitude of this stabilization is similar to the extra stabilization of $\mathrm{O}_{\text {ads }}$ as compared to the adsorbates involving the attraction of a single electron.

\subsection{Energetics of the Full OER}

With the solvent effect on the individual PCET steps of eq. (9) analyzed, we focus on the full reaction mechanism in eq. (1). We initially consider a bare active site on which a water molecule adsorbs. The adsorption free energy $\Delta F_{\text {ads }}\left[\mathrm{H}_{2} \mathrm{O}\right]$ of a liquid $\mathrm{H}_{2} \mathrm{O}$ molecule is added to the first free energy step. The final reaction step involves the desorption of the oxygen molecule, resulting in the free energy term $\Delta F_{\text {des }}\left[\mathrm{O}_{2}\right]$. Here, we evaluate these free energies through the CHE framework, as this method has been found above to give accurate adsorption free energies when the described system does not involve notable charging. In this way, we find that the free energy of $\mathrm{H}_{2} \mathrm{O}$ adsorption $\Delta F_{\text {ads }}\left[\mathrm{H}_{2} \mathrm{O}\right]=-0.37 \mathrm{eV}$, and the free energy of $\mathrm{O}_{2}$ desorption $\Delta F_{\text {des }}\left[\mathrm{O}_{2}\right]=-1.30 \mathrm{eV}$. Furthermore, in the third step, we need to evaluate the free energy $\Delta F\left[\mathrm{O}_{\text {ads }} \rightarrow \mathrm{H}_{2} \mathrm{O}_{2}\right.$ ads $]$ associated with the formation of $\mathrm{H}_{2} \mathrm{O}_{2}$ ads (see insets in Fig. 4). In this reaction, the charge attracted by the adsorbates drops from 
$-2 e$ to $-e$. To evaluate the corresponding free energy difference, we use the Blue Moon method, as described in sec. 2.4.

Figure 4 shows the calculated free energy profiles for the solvated and the vacuum case, for which we find free energy differences of $-0.14 \pm 0.02 \mathrm{eV}$ and $-0.69 \pm 0.03 \mathrm{eV}$, respectively. The difference of $0.55 \mathrm{eV}$ in the values of $\Delta F\left[\mathrm{O}_{\text {ads }} \rightarrow \mathrm{H}_{2} \mathrm{O}_{2}\right.$ ads $]$ can be attributed to the different screening conditions in the two cases, and is quantitatively consistent with the results found in Fig. 2. For comparison, we also evaluate this free energy difference within the CHE approach and find $\Delta F\left[\mathrm{O}_{\text {ads }} \rightarrow \mathrm{H}_{2} \mathrm{O}_{2}\right.$ ads $]=-0.88 \mathrm{eV}$, in good agreement with our Blue Moon results for the vacuum interface. With the above additions, we calculate the OER free energy steps as follows:

$$
\begin{aligned}
& \Delta G_{1}=\Delta G_{1}^{\prime}+\Delta F_{\text {ads }}\left[\mathrm{H}_{2} \mathrm{O}\right], \\
& \Delta G_{2}=\Delta G_{2}^{\prime}, \\
& \Delta G_{3}=\Delta G_{3}^{\prime}+\Delta F\left[\mathrm{O}_{\mathrm{ads}} \rightarrow \mathrm{H}_{2} \mathrm{O}_{2 \text { ads }}\right], \\
& \Delta G_{4}=\Delta G_{4}^{\prime}+\Delta F_{\text {des }}\left[\mathrm{O}_{2}\right] .
\end{aligned}
$$

Table 4: Free energy steps $\Delta G_{i}$ calculated using the thermodynamic integration (TI) method with and without solvent, and the computational hydrogen electrode (CHE) method. All values are in $\mathrm{eV}$.

\begin{tabular}{lcrc}
\hline \hline & CHE & TI no solvent & TI solvent \\
\hline$\Delta G_{1}$ & 1.39 & $1.29 \pm 0.11$ & $1.29 \pm 0.17$ \\
$\Delta G_{2}$ & 2.43 & $2.36 \pm 0.05$ & $1.82 \pm 0.06$ \\
$\Delta G_{3}$ & 0.24 & $0.53 \pm 0.06$ & $1.11 \pm 0.07$ \\
$\Delta G_{4}$ & 0.08 & $-0.26 \pm 0.08$ & $-0.22 \pm 0.11$ \\
\hline \hline
\end{tabular}

The computed OER free energy steps are given in Table 4 and are illustrated in Fig. 5. Note that the sum of all $\Delta G$ steps attains a value of about $4.0 \mathrm{eV}$ regardless of the method used. Indeed, due to the catalytic effect of the substrate, the sum of the OER free energy steps should yield a single value independent of the method, provided the initial and final states are described in the same way. In this sense, our results are reassuring because they 


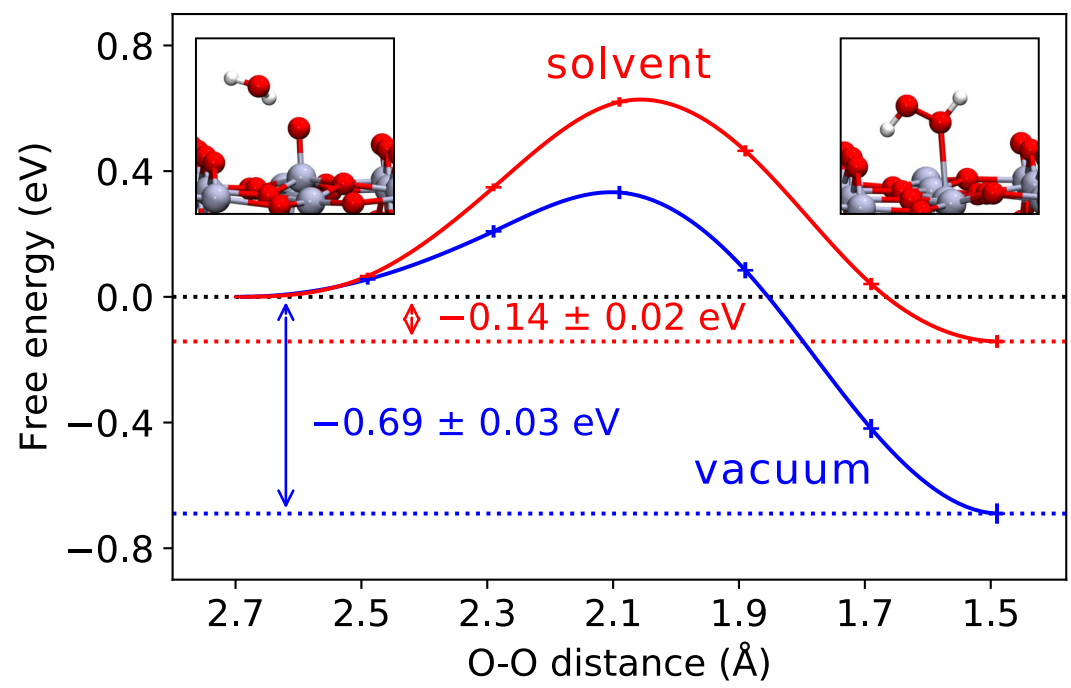

Figure 4: Free energy profile of the $\mathrm{H}_{2} \mathrm{O}_{2}$ ads formation in the vacuum (blue) and in the solvated (red) case. The insets illustrate the initial and final configurations. The solvent is not shown for clarity.

all yield a consistent free energy sum within less than $0.15 \mathrm{eV}$. Unlike in other work, ${ }^{24,28,37}$ we do not adjust the free energy of the last OER step and thus record a discrepancy with the experimental free energy for the full OER reaction, $\Delta G_{\mathrm{OER}}=4.92 \mathrm{eV} .{ }^{58}$ The difference between the calculated and measured $\Delta G_{\text {OER }}$ reflects the limitations of the adopted semilocal energy functional. Indeed, as can be tested with the CHE approach, the use of a hybrid functional with $\alpha=0.40$ augmented with van der Waals corrections, which successfully describes the properties of liquid water, ${ }^{46}$ leads to a calculated $\Delta G_{\text {OER }}=4.84 \mathrm{eV}$, in much better agreement with the experimental value.

\section{Discussion}

The presence of explicit water molecules has a noticeable influence on the $\Delta G$ values of the OER. First, the reaction determining step $\Delta G_{2}$ of the OER at the $\mathrm{TiO}_{2}$-vacuum interface is reduced by $0.5 \mathrm{eV}$ upon inclusion of the solvent. In the work of Cheng et al. ${ }^{25}$ only a fraction of a water monolayer is used to model the solvent. The effect of hydrogen bond 

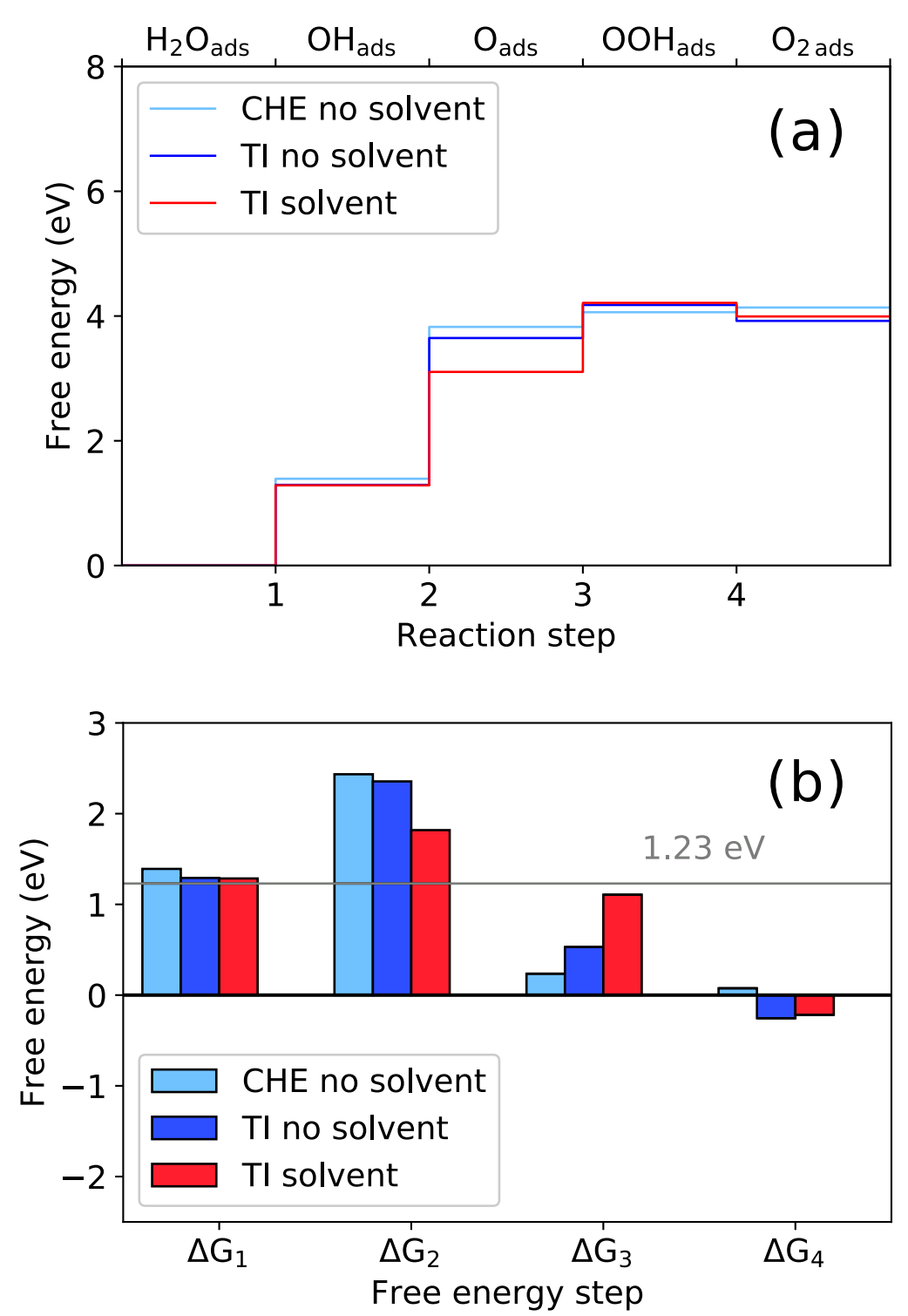

Figure 5: Comparison of the free energies corresponding to the full OER mechanism calculated using the CHE method and the TI method.

stabilization and charge effects are therefore not fully captured and their result of $2.4 \mathrm{eV}$ might be overestimated, as can be inferred by comparing our values of $\Delta G_{2}$ with and without solvent. Second, the stabilization of the $\mathrm{O}_{\mathrm{ads}}$ intermediate in the presence of liquid water leads to an increase of the third free energy step $\Delta G_{3}$. This contrasts with results from static calculations at the similar $\mathrm{IrO}_{2}$ interface in which the solvation effect was found to yield reduced overpotentials in the first three OER steps. ${ }^{37}$ This difference most likely results 
from the consideration of a relaxed configuration for $\mathrm{O}_{\text {ads }}$ that differs from the equilibrium structure that we observe in the molecular dynamics simulations. For the same reason, in the CHE approach, our value of $\Delta G_{2}$ is noticeably larger than that found by Valdés et al. $(1.47 \mathrm{eV}) \cdot{ }^{24}$

\section{Conclusion}

We described the solvent effects on the energetics of the OER at finite temperature. In the absence of the solvent, the computational hydrogen electrode method yields free energies that agree well with those obtained by thermodynamic integration. A significant reduction of the free energy associated with the rate determining step is observed upon inclusion of the solvent. An analysis of the structure at the solvated interface, with a particular focus on hydrogen bonds, is insufficient to explain the observed trends. Instead, the large stabilization of the oxygen intermediate due to the solvent can be understood in terms of the screening at the interface. This finding supports the use of implicit models for describing the solvent, which generally capture the electrostatics but are unable to account for hydrogen bonds. In the light of the present study, it is recommended that solvent effects be carefully considered in ongoing searches for novel OER photocatalysts.

\section{Acknowledgement}

This work has been realized in relation to the National Center of Competence in Research (NCCR) "Materials' Revolution: Computational Design and Discovery of Novel Materials (MARVEL)" of the SNSF. We used computational resources of the Swiss National Supercom-

puting Centre (CSCS) and of the Scientific IT and Application Support Center (SCITAS) of Ecole Polytechnique Fédérale de Lausanne. 


\section{References}

(1) Seh, Z. W.; Kibsgaard, J.; Dickens, C. F.; Chorkendorff, I.; Nørskov, J. K.; Jaramillo, T. F. Combining Theory and Experiment in Electrocatalysis: Insights into Materials Design. Science 2017, 355, eaad4998.

(2) McCrory, C. C. L.; Jung, S.; Peters, J. C.; Jaramillo, T. F. Benchmarking Heterogeneous Electrocatalysts for the Oxygen Evolution Reaction. J. Am. Chem. Soc. 2013, 135, $16977-16987$.

(3) Walter, M. G.; Warren, E. L.; McKone, J. R.; Boettcher, S. W.; Mi, Q.; Santori, E. A.; Lewis, N. S. Solar Water Splitting Cells. Chem. Rev. 2010, 110, 6446-6473.

(4) Tentu, R. D.; Basu, S. Photocatalytic Water Splitting for Hydrogen Production. Curr. Opin. Electrochem. 2017, 5, 56-62.

(5) Suen, N.-T.; Hung, S.-F.; Quan, Q.; Zhang, N.; Xu, Y.-J.; Ming Chen, H. Electrocatalysis for the Oxygen Evolution Reaction: Recent Development and Future Perspectives. Chem. Soc. Rev. 2017, 46, 337-365.

(6) Montoya, J. H.; Seitz, L. C.; Chakthranont, P.; Vojvodic, A.; Jaramillo, T. F.; Nørskov, J. K. Materials for Solar Fuels and Chemicals. Nature 2017, 16, 70-81.

(7) Fujishima, A.; Honda, K. Electrochemical Photolysis of Water at a Semiconductor Electrode. Nature 1972, 238, 37.

(8) Hashimoto, K.; Irie, H.; Fujishima, A. $\mathrm{TiO}_{2}$ Photocatalysis: A Historical Overview and Future Prospects. J. Appl. Phys. 2005, 44, 8269.

(9) Chen, J.; Li, Y.-F.; Sit, P.; Selloni, A. Chemical Dynamics of the First Proton-Coupled Electron Transfer of Water Oxidation on $\mathrm{TiO}_{2}$ Anatase. J. Am. Chem. Soc. 2013, 135, $18774-18777$. 
(10) Selcuk, S.; Selloni, A. Facet-Dependent Trapping and Dynamics of Excess Electrons at Anatase $\mathrm{TiO}_{2}$ Surfaces and Aqueous Interfaces. Nature 2016, 15, 1107-1112.

(11) Savory, D. M.; McQuillan, A. J. IR Spectroscopic Behavior of Polaronic Trapped Electrons in $\mathrm{TiO}_{2}$ Under Aqueous Photocatalytic Conditions. J. Phys. Chem. 2014, 118, $13680-13692$.

(12) Di Valentin, C.; Fittipaldi, D. Hole Scavenging by Organic Adsorbates on the $\mathrm{TiO}_{2}$ Surface: A DFT Model Study. Chem. Lett. 2013, 4, 1901-1906.

(13) Li, Y.-F.; Liu, Z.-P.; Liu, L.; Gao, W. Mechanism and Activity of Photocatalytic Oxygen Evolution on Titania Anatase in Aqueous Surroundings. J. Am. Chem. Soc. 2010, 132, 13008-13015.

(14) Stecher, T.; Reuter, K.; Oberhofer, H. First-Principles Free-Energy Barriers for Photoelectrochemical Surface Reactions: Proton Abstraction at $\mathrm{TiO}_{2}(110)$. Phys. Rev. Lett. 2016, 117, 276001.

(15) Zhang, D.; Yang, M.; Dong, S. Hydroxylation of the Rutile $\mathrm{TiO}_{2}$ (110) Surface Enhancing Its Reducing Power for Photocatalysis. J. Phys. Chem. 2015, 119, 1451-1456.

(16) Deskins, N. A.; Rousseau, R.; Dupuis, M. Localized Electronic States from Surface Hydroxyls and Polarons in $\mathrm{TiO}_{2}(110)$. J. Phys. Chem. 2009, 113, 14583-14586.

(17) Deskins, N. A.; Rousseau, R.; Dupuis, M. Defining the Role of Excess Electrons in the Surface Chemistry of $\mathrm{TiO}_{2}$. J. Phys. Chem. 2010, 114, 5891-5897.

(18) Deskins, N. A.; Rousseau, R.; Dupuis, M. Distribution of $\mathrm{Ti}^{3+}$ Surface Sites in Reduced $\mathrm{TiO}_{2}$. J. Phys. Chem. 2011, 115, 7562-7572.

(19) Nakamura, R.; Nakato, Y. Primary Intermediates of Oxygen Photoevolution Reaction on $\mathrm{TiO}_{2}$ (Rutile) Particles, Revealed by in Situ FTIR Absorption and Photoluminescence Measurements. J. Am. Chem. Soc. 2004, 126, 1290-1298. 
(20) Di Valentin, C.; Pacchioni, G.; Selloni, A. Electronic Structure of Defect States in Hydroxylated and Reduced Rutile $\mathrm{TiO}_{2}$ (110) Surfaces. Phys. Rev. Lett. 2006, 97, 166803.

(21) Shibuya, T.; Yasuoka, K.; Mirbt, S.; Sanyal, B. A Systematic Study of Polarons Due to Oxygen Vacancy Formation at the Rutile $\mathrm{TiO}_{2}$ (110) Surface by GGA + U and HSE06 Methods. J. Phys.: Condens. Matter 2012, 24, 435504.

(22) Chrétien, S.; Metiu, H. Electronic Structure of Partially Reduced Rutile $\mathrm{TiO}_{2}(110)$ Surface: Where Are the Unpaired Electrons Located? J. Phys. Chem. 2011, 115, 4696-4705.

(23) Cheng, J.; Sprik, M. Aligning Electronic Energy Levels at the $\mathrm{TiO}_{2} / \mathrm{H}_{2} \mathrm{O}$ Interface. Phys. Rev. B 2010, 82, 081406.

(24) Valdés, Á.; Qu, Z.-W.; Kroes, G.-J.; Rossmeisl, J.; Nørskov, J. K. Oxidation and PhotoOxidation of Water on $\mathrm{TiO}_{2}$ Surface. J. Phys. Chem. 2008, 112, 9872-9879.

(25) Cheng, J.; Liu, X.; Kattirtzi, J. A.; VandeVondele, J.; Sprik, M. Aligning Electronic and Protonic Energy Levels of Proton-Coupled Electron Transfer in Water Oxidation on Aqueous $\mathrm{TiO}_{2}$. Angew. Chem. Int. Ed. 2014, 53, 12046-12050.

(26) Liu, S.; White, M. G.; Liu, P. Mechanism of Oxygen Reduction Reaction on Pt(111) in Alkaline Solution: Importance of Chemisorbed Water on Surface. J. Phys. Chem. C 2016, 120, 15288-15298.

(27) Oberhofer, H. In Handbook of Materials Modeling: Applications: Current and Emerging Materials; Andreoni, W., Yip, S., Eds.; Springer International Publishing: Cham, 2018; pp 1-33.

(28) Nørskov, J. K.; Rossmeisl, J.; Logadottir, A.; Lindqvist, L.; Kitchin, J. R.; Bligaard, T.; 
Jónsson, H. Origin of the Overpotential for Oxygen Reduction at a Fuel-Cell Cathode. J. Phys. Chem. 2004, 108, 17886-17892.

(29) A. Peterson, A.; Abild-Pedersen, F.; Studt, F.; Rossmeisl, J.; K. Nørskov, J. How Copper Catalyzes the Electroreduction of Carbon Dioxide into Hydrocarbon Fuels. Energy Environ. 2010, 3, 1311-1315.

(30) Ambrosio, F.; Wiktor, J.; Pasquarello, A. pH-Dependent Surface Chemistry from First Principles: Application to the BiVO4(010)-Water Interface. ACS Appl. Mater. Interfaces 2018, 10, 10011-10021.

(31) Man, I. C.; Su, H.-Y.; Calle-Vallejo, F.; Hansen, H. A.; Martínez, J. I.; Inoglu, N. G.; Kitchin, J.; Jaramillo, T. F.; Nørskov, J. K.; Rossmeisl, J. Universality in Oxygen Evolution Electrocatalysis on Oxide Surfaces. ChemCatChem 2011, 3, 1159-1165.

(32) Sha, Y.; Yu, T. H.; Liu, Y.; Merinov, B. V.; Goddard, W. A. Theoretical Study of Solvent Effects on the Platinum-Catalyzed Oxygen Reduction Reaction. J. Phys. Chem. Lett. 2010, 1, 856-861.

(33) Fortunelli, A.; Iii, W. A. G.; Sementa, L.; Barcaro, G. Optimizing the Oxygen Evolution Reaction for Electrochemical Water Oxidation by Tuning Solvent Properties. Nanoscale 2015, 7, 4514-4521.

(34) Ping, Y.; Nielsen, R. J.; Goddard, W. A. The Reaction Mechanism with Free Energy Barriers at Constant Potentials for the Oxygen Evolution Reaction at the IrO2 (110) Surface. J. Am. Chem. Soc. 2017, 139, 149-155.

(35) Skúlason, E.; Karlberg, G. S.; Rossmeisl, J.; Bligaard, T.; Greeley, J.; Jónsson, H.; Nørskov, J. K. Density Functional Theory Calculations for the Hydrogen Evolution Reaction in an Electrochemical Double Layer on the Pt(111) Electrode. Phys. Chem. Chem. Phys. 2007, 9, 3241-3250. 
(36) Skúlason, E.; Tripkovic, V.; Björketun, M. E.; Gudmundsdóttir, S.; Karlberg, G.; Rossmeisl, J.; Bligaard, T.; Jónsson, H.; Nørskov, J. K. Modeling the Electrochemical Hydrogen Oxidation and Evolution Reactions on the Basis of Density Functional Theory Calculations. J. Phys. Chem. C 2010, 114, 18182-18197.

(37) Gauthier, J. A.; Dickens, C. F.; Chen, L. D.; Doyle, A. D.; Nørskov, J. K. Solvation Effects for Oxygen Evolution Reaction Catalysis on IrO2(110). J. Phys. Chem. 2017, 121, 11455-11463.

(38) Hansen, M. H.; Rossmeisl, J. pH in Grand Canonical Statistics of an Electrochemical Interface. J. Phys. Chem. C 2016, 120, 29135-29143.

(39) Ludwig, T.; Gauthier, J. A.; Brown, K. S.; Ringe, S.; Nørskov, J. K.; Chan, K. Solvent-Adsorbate Interactions and Adsorbate-Specific Solvent Structure in Carbon Dioxide Reduction on a Stepped Cu Surface. J. Phys. Chem. C 2019, 123, 5999-6009.

(40) Frenkel, D.; Smit, B. In Understanding Molecular Simulation (Second Edition), second edition ed.; Frenkel, D., Smit, B., Eds.; Academic Press: San Diego, 2002; pp 167 200.

(41) Costanzo, F.; Sulpizi, M.; Valle, R. G. D.; Sprik, M. The Oxidation of Tyrosine and Tryptophan Studied by a Molecular Dynamics Normal Hydrogen Electrode. J. Chem. Phys. 2011, 134, 244508.

(42) Patel, A. M.; Ringe, S.; Siahrostami, S.; Bajdich, M.; Nørskov, J. K.; Kulkarni, A. R. Theoretical Approaches to Describing the Oxygen Reduction Reaction Activity of Single-Atom Catalysts. J. Phys. Chem. C 2018, 122, 29307-29318.

(43) Hutter, J.; Iannuzzi, M.; Schiffmann, F.; VandeVondele, J. CP2K: Atomistic Simulations of Condensed Matter Systems. Science 2014, 4, 15-25. 
(44) Sabatini, R.; Gorni, T.; de Gironcoli, S. Nonlocal Van Der Waals Density Functional Made Simple and Efficient. Phys. Rev. B 2013, 87, 041108.

(45) Miceli, G.; de Gironcoli, S.; Pasquarello, A. Isobaric First-Principles Molecular Dynamics of Liquid Water with Nonlocal Van Der Waals Interactions. Chem. Phys. 2015, 142, 034501 .

(46) Ambrosio, F.; Miceli, G.; Pasquarello, A. Redox Levels in Aqueous Solution: Effect of Van Der Waals Interactions and Hybrid Functionals. Chem. Phys. 2015, 143, 244508.

(47) Oberhofer, H.; Reuter, K. First-Principles Thermodynamic Screening Approach to Photo-Catalytic Water Splitting with Co-Catalysts. Chem. Phys. 2013, 139, 044710.

(48) Gono, P.; Wiktor, J.; Ambrosio, F.; Pasquarello, A. Surface Polarons Reducing Overpotentials in the Oxygen Evolution Reaction. ACS Catal. 2018, 8, 5847-5851.

(49) Goedecker, S.; Teter, M.; Hutter, J. Separable Dual-Space Gaussian Pseudopotentials. Phys. Rev. B 1996, 54, 1703-1710.

(50) VandeVondele, J.; Mohamed, F.; Krack, M.; Hutter, J.; Sprik, M.; Parrinello, M. The Influence of Temperature and Density Functional Models in Ab Initio Molecular Dynamics Simulation of Liquid Water. Chem. Phys. 2004, 122, 014515.

(51) Fujii, K. In Solar to Chemical Energy Conversion: Theory and Application; Sugiyama, M., Fujii, K., Nakamura, S., Eds.; Springer International Publishing: Cham, 2016; pp 7-34.

(52) Cheng, J.; Sprik, M. Alignment of Electronic Energy Levels at Electrochemical Interfaces. Phys. Chem. Chem. Phys. 2012, 14, 11245-11267.

(53) Flyvbjerg, H. Advances in Computer Simulation, 1st ed.; Lect. Notes Phys.; Springer, Berlin, Heidelberg, 1998; Vol. 501; pp 88-103. 
(54) Soper, A. K.; Bruni, F.; Ricci, M. A. Site-Site Pair Correlation Functions of Water from 25 to $400{ }^{\circ} \mathrm{C}$ : Revised Analysis of New and Old Diffraction Data. J. Chem. Phys. 1997, 106, 247-254.

(55) Komsa, H.-P.; Pasquarello, A. Finite-Size Supercell Correction for Charged Defects at Surfaces and Interfaces. Phys. Rev. Lett. 2013, 110, 095505.

(56) Sprik, M.; Ciccotti, G. Free Energy from Constrained Molecular Dynamics. Chem. Phys. 1998, 109, 7737-7744.

(57) Ciccotti, G.; Ferrario, M. Rare Events by Constrained Molecular Dynamics. J. Mol. Liq. 2000, 89, 1-18.

(58) Dau, H.; Limberg, C.; Reier, T.; Risch, M.; Roggan, S.; Strasser, P. The Mechanism of Water Oxidation: From Electrolysis via Homogeneous to Biological Catalysis. ChemCatChem 2010, 2, 724-761. 


\section{TOC Graphic}

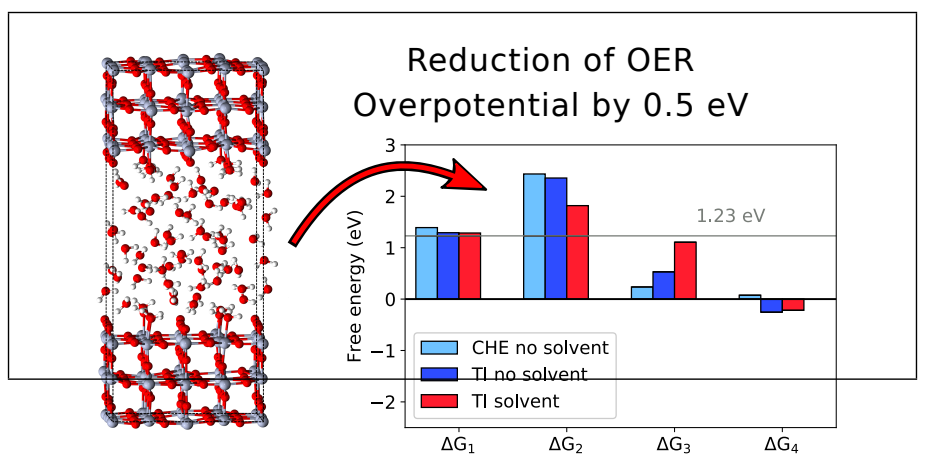

